\title{
Direct healthcare cost comparison of Fluticasone propionate/Salmeterol vs Budesonide/Formoterol Maintenance And Reliever Therapy for moderate/severe asthma: Results from Thailand
}

\author{
Torsak Bunupuradah, ${ }^{1}$ Watchara Boonsawat, ${ }^{2}$ Jatupum Kamrapit, ${ }^{1}$ Bhumika Aggarwal ${ }^{3}$
}

\begin{abstract}
Background: Inhaled corticosteroid/long-acting $\beta 2$-agonist (ICS/LABA) for moderate/severe asthma i.e. regular Fluticasone propionate/Salmeterol (FP/Salm) with as-needed short acting beta-2 agonist (SABA) or ICS/Formoterol Maintenance And Reliever Therapy (MART) are the recommended options.
\end{abstract}

Objective: To compare healthcare cost between regular FP/Salm with as-needed SABA vs MART in Thailand.

Methods: Direct healthcare cost data from 3 published randomized trials in asthma patients aged $\geq 12$ years comparing regular twice-daily FP/Salm with as-needed SABA vs Budesonide/Formoterol (BUD/Form) MART in moderate/severe asthma were considered: AHEAD (NCT00242775/17 countries/2309 patients), COMPASS (AstraZeneca study SD-0390735/16 countries/3335 patients), and COSMOS (AstraZeneca study SD-039-0691/16 countries/2143 patients). Total direct treatment cost comparison/patient/year was calculated as a combination from 1) medication costs plus 2) healthcare utilization costs i.e. cost for health care visit, emergency room visit, and hospitalization. Unit costs referred from National drug information and Health Intervention and Technology Assessment (HITAP), Ministry of Public Health.

Results: Annual medication costs of FP/Salm + SABA were lower than MART in all studies with average cost as 182.01 vs 347.21 USD. Average annual healthcare utilization costs were 17.51 vs 13.01 USD in FP/Salm + SABA and MART, respectively. In overall, total direct treatment costs/patient/year with FP/Salm was 199.53 vs 360.22 USD of MART. Percent saving of total direct treatment costs by FP/Salm + SABA was $45 \%$ lower than with MART.

Conclusion: In moderate/severe asthma patients, total direct treatment costs with regular twice-daily FP/Salm with as-needed SABA were lower than with BUD/Form MART primarily due to lower medication costs. Healthcare cost should be considered for asthma care in Thailand.

Key words: Asthma, Fluticasone propionate/Salmeterol, Budesonide/Formoterol Maintenance And Reliever Therapy, MART, ICS/LABA, Thailand

From:

${ }^{1}$ GlaxoSmithKline Limited, Bangkok, Thailand

2 Department of Medicine, Faculty of Medicine, Khon Kaen University,

Khon Kaen, Thailand

GlaxoSmithKline Limited, Singapore

Corresponding author:

Torsak Bunupuradah

$12^{\text {th }}$ Floor, Wave Place, 55 Wireless Road, Lumpini, Patumwan,

Bangkok 10330, Thailand

E-mail: torsak.x.bunupuradah@gsk.com

\section{Introduction}

Asthma is a common chronic airway inflammatory respiratory disease affecting $1-18 \%$ of population. ${ }^{1}$ Prevalence of asthma in Thailand was $8.8-9.8 \% .^{2,3}$ Asthma has high burden in Thailand as $14.8 \%$ of adults with asthma had been hospitalized. ${ }^{4}$ The main asthma treatment is daily anti-inflammatory agents to achieve asthma control and prevent future risks. ${ }^{1}$ Commonly used anti-inflammatory agents are inhaled corticosteroids (ICS) and inhaled corticosteroids/long acting beta- 2 agonists (ICS/LABA). ${ }^{1}$ Patients with moderatesevere asthma symptoms can be initiated with ICS/LABA. ${ }^{1}$ 
Step-up from regular ICS to regular ICS/LABA is recommended for poorly controlled asthma patients. ${ }^{1}$

Stepwise treatment approach for asthma patients using ICS/LABA demonstrated higher efficacy than ICS alone. ${ }^{5}$ From The Gaining Optimal Asthma ControL Study (GOAL study), patients randomized to Fluticasone propionate/Salmeterol (FP/Salm) achieved significantly greater well-controlled and total-controlled week, shorter duration to achieve control, and used less ICS dose than Fluticasone Propionate (FP) alone. ${ }^{5}$ Overall, $71 \%$ of FP/Salm group became well-controlled at 1 year. $^{5}$

Budesonide/Formoterol Maintenance And Reliever Therapy (MART) is also recommended for moderate/severe asthma. ${ }^{1,6}$ MART relies on rapid as-needed adjustments in ICS/ LABA with the aim of reducing severe exacerbations. Although MART had lower severe asthma exacerbation but less than one-fifth of patients with asthma on MART regimen had control as per GINA criteria. ${ }^{7,8}$

Asthma can cause significant economic burden. ${ }^{9}$ The asthma treatment costs are variable from country to country. Previous studies comparing cost between FP/Salm vs MART were from resource-rich countries. ${ }^{10-12}$ There is limited data comparing treatment cost between both strategies in resource limited setting. ${ }^{13}$ Therefore, we compared health care cost based from three randomized control trials of regular FP/Salm plus as needed SABA vs MART ${ }^{14-16}$ by using current available data of medication cost and healthcare utilization costs in Thailand.

\section{Methods}

From a targeted literature review using PubMed, there were 3 published randomized trials in asthma patients aged $\geq 12$ years comparing regular twice-daily FP/Salm with as-needed SABA vs MART in moderate/severe asthma: AHEAD (NCT00242775/17 countries/2309 patients), ${ }^{14}$ COMPASS (AstraZeneca study SD-039-0735/16 countries/3335 patients), ${ }^{15}$ and COSMOS (AstraZeneca study SD-039-0691/16 countries/2143 patients). ${ }^{16}$
In brief, $\mathrm{AHEAD}$ is a 6-month, randomized, double-blind, parallel group, comparing FP/Salm $(500 / 50 \mu \mathrm{g}) 1$ inhalation bid + terbutaline as needed vs BUD/Form $(160 / 4.5 \mu \mathrm{g})$ 2 inhalation bid + BUD/Form as needed. ${ }^{14}$ COMPASS is a 6-month, randomized, double-blind, double dummy, parallel group comparing FP/Salm (125/25) $\mu \mathrm{g} 2$ inhalation bid + terbutaline as needed vs BUD/Form (320/9 $\mu \mathrm{g}) 1$ inhalation bid + terbutaline as needed (data from this study arm was not included in this analysis) vs BUD/Form (160/4.5 $\mu \mathrm{g}) 1$ inhalation bid + BUD/Form as needed. ${ }^{15}$ COSMOS is a 12 -month, randomized, open-label, parallel group comparing FP/Salm $(250 / 50 \mu \mathrm{g}) 1$ inhalation bid (dose could be changed to $100 / 50$ $\mu \mathrm{g} \times 1$ inhalation bid or $500 / 50 \mu \mathrm{g} \times 1$ inhalation bid $)+$ salbutamol as needed vs BUD/Form (160/4.5 $\mu \mathrm{g}) 2$ inhalations bid (dose could be changed to $160 / 4.5 \mu \mathrm{g} \times 1$ inhalations bid) + BUD/Form as needed. ${ }^{16}$ These 3 studies had the same primary endpoint as time to first severe exacerbation. ${ }^{14-16}$ In overall, time to first severe asthma exacerbation was significantly prolonged in patients using MART compared with FP/ Salm in the COMPASS ${ }^{15}$ and COSMOS ${ }^{16}$ studies but had no significant difference in AHEAD study. ${ }^{14}$

\section{Number of resource use per patient}

During all studies, resource utilization data were collected via patient notebooks or event logs including hospitalization, emergency room visits, healthcare visits for general practitioner and specialist, nurse, and physiotherapist. ${ }^{11,14-16}$ For comparative purposes, data from 6-month studies, AHEAD ${ }^{14}$ and COMPASS, ${ }^{15}$ were extrapolated with 12 months. ${ }^{17}$ Mean numbers of resource use per patient by studies and by treatment arms are shown in Table $\mathbf{1}$.

\section{Economic Analysis}

Total direct treatment cost comparison/patient/year was calculated as a combination from 1) medication costs plus 2) healthcare utilization costs i.e. cost for health care visit, emergency room visit, and hospitalization. ${ }^{17}$ Medication costs were referred from Thailand median price 2020, National drug

Table 1. Mean number of resource use per patient by studies

\begin{tabular}{|c|c|c|c|c|c|c|}
\hline & \multicolumn{2}{|c|}{ AHEAD $^{11,14}$} & \multicolumn{2}{|c|}{ COMPASS $^{11,15}$} & \multicolumn{2}{|c|}{$\operatorname{COSMOS}^{11,16}$} \\
\hline & FP/Salm & MART & FP/Salm & MART & FP/Salm & MART \\
\hline \multicolumn{7}{|l|}{ Medication use/patient/day } \\
\hline 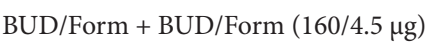 & - & 4.879 & - & 3.250 & - & 3.94 \\
\hline BUD/Form $(320 / 9 \mu \mathrm{g})$ & - & - & - & - & - & - \\
\hline FP/Salm $(125 / 25 \mu \mathrm{g})$ & - & - & 4.304 & - & - & - \\
\hline FP/Salm $(100 / 50 \mu \mathrm{g})$ & - & - & - & - & 0.233 & - \\
\hline FP/Salm $(250 / 50 \mu \mathrm{g})$ & - & - & - & - & 1.214 & - \\
\hline FP/Salm $(500 / 50 \mu \mathrm{g})$ & 1.978 & - & - & - & 0.490 & - \\
\hline Salbutamol & - & - & - & - & 0.907 & - \\
\hline Terbutaline $(0.4 \mathrm{mg})$ & 0.995 & - & 1.036 & - & - & - \\
\hline
\end{tabular}


Table 1. (Continued)

\begin{tabular}{|c|c|c|c|c|c|c|}
\hline & \multicolumn{2}{|c|}{ AHEAD $^{11,14}$} & \multicolumn{2}{|c|}{ COMPASS $^{11,15}$} & \multicolumn{2}{|c|}{$\operatorname{cosMOS}^{11,16}$} \\
\hline & FP/Salm & MART & FP/Salm & MART & FP/Salm & MART \\
\hline \multicolumn{7}{|l|}{ Healthcare use/patient/year } \\
\hline \multicolumn{7}{|l|}{ Hospitalization } \\
\hline Intensive care unit (ICU) & 0 & 0.01 & 0.012 & 0.048 & 0.005 & 0.009 \\
\hline General ward & 0.05 & 0.06 & 0.296 & 0.074 & 0.09 & 0.05 \\
\hline \multicolumn{7}{|l|}{ Healthcare visit } \\
\hline Emergency department & 0.104 & 0.094 & 0.178 & 0.134 & 0.06 & 0.04 \\
\hline Specialist & 0.240 & 0.152 & 0.408 & 0.314 & 0.24 & 0.17 \\
\hline General practitioner & 0.192 & 0.168 & 0.270 & 0.282 & 0.37 & 0.32 \\
\hline Other & 0.056 & 0.028 & 0.096 & 0.074 & 0.10 & 0.05 \\
\hline \multicolumn{7}{|l|}{ Home visit } \\
\hline General practitioner & 0.016 & 0.010 & 0.016 & 0.006 & 0.04 & 0.03 \\
\hline Other & 0.006 & 0.002 & 0.044 & 0.006 & 0.01 & 0 \\
\hline
\end{tabular}

Data for AHEAD and COMPASS studies extrapolated to 1 year.

BUD/Form: Budesonide/Formoterol, FP/Salm: Fluticasone propionate/Salmeterol

Table 2. Cost of resource utilization

\begin{tabular}{lcc}
\multicolumn{1}{|c}{ Items of resource utilization } & Thai Baht & USD \\
\hline Medication cost, ${ }^{13}$ per patient per dose/puff & & \\
\hline BUD/Form + BUD/Form $(160 / 4.5 \mu \mathrm{g})$ & 7.42 & 0.24 \\
\hline BUD/Form $(320 / 9 \mu \mathrm{g})$ & 10.52 & 0.34 \\
\hline FP/Salm $(125 / 25 \mu \mathrm{g})$ & 2.50 & 0.08 \\
\hline FP/Salm $(100 / 50 \mu \mathrm{g})$ & 6.42 & 0.21 \\
\hline FP/Salm $(250 / 50 \mu \mathrm{g})$ & 7.79 & 0.25 \\
\hline FP/Salm $(500 / 50 \mu \mathrm{g})$ & 9.95 & 0.32 \\
\hline Salbutamol & 0.23 & 0.01 \\
\hline Terbutaline $(0.4 \mathrm{mg})$ & 0.23 & 0.01 \\
\hline Healthcare utilization costs & & \\
\hline Hospitalization, ${ }^{13}$ per day & & \\
\hline Intensive care unit (ICU) & 5,070 & 162.04 \\
\hline General ward & 1,953 & 62.42 \\
\hline Healthcare visit, ${ }^{19}$ per visit & & \\
\hline Emergency room ${ }^{35}$ & 118 & 3.77 \\
\hline Specialist & 396.36 & 25.45 \\
\hline General care physician & 306 & 9.78 \\
\hline Other & 359.3 & 11.48 \\
\hline Home visit, ${ }^{19}$ per visit & & 3.23 \\
\hline General care physician & & \\
\hline Other & & \\
\hline BD/For & & \\
\hline
\end{tabular}

information. ${ }^{18}$ Cost of medications were based on the licensed dose and calculated and converted to United States of America Dollars (USD)/dose or USD/puff. Healthcare utilization costs were referred from Health Intervention and Technology Assessment (HITAP), Ministry of Public Health. ${ }^{19}$ Unit cost of resource utilization are shown in Table 2 . Percent saving total cost by FP/Salm was calculated by [(total treatment cost MART - total treatment cost FP/Salm)/total treatment cost MART] $\times 100$.

\section{Results}

Medication, healthcare utilization, and total direct treatment cost by studies as USD/patient/year across the 3 studies and per treatment arms were shown in Table 3. Annual medication costs of twice-daily regular dosing with FP/Salm + as-needed SABA were lower than BUD/Form MART in all three studies with average cost as 182.01 vs 347.21 USD/ patient/year. Average annual healthcare utilization costs were 17.51 vs $13.01 \mathrm{USD} /$ patient/year in twice-daily regular dosing with $\mathrm{FP} / \mathrm{Salm}+$ as-needed SABA were lower than BUD/Form MART, respectively. Overall, total direct treatment costs in case of twice-daily regular dosing with FP/Salm was 199.53 vs 360.22 USD/patient/year of BUD/Form MART. Percent saving of total direct treatment costs by twice-daily regular dosing with $\mathrm{FP} / \mathrm{Salm} \mathrm{FP} / \mathrm{Salm}+$ as-needed SABA was $45 \%$ lower than with BUD/Form MART. Total direct treatment cost by treatment options in each study are shown in Figure $\mathbf{1}$.

BUD/Form: Budesonide/Formoterol; FP/Salm: Fluticasone propionate/Salmetero Note: 1 Thai Baht $=0.03196$ USD (UNCTADstat annual currency exchange rates; 2020) 
Table 3. Annual medication, healthcare utilization, and total direct treatment cost by studies

\begin{tabular}{|lccccc|}
\hline & $\begin{array}{c}\text { Treatment } \\
\text { arm }\end{array}$ & $\begin{array}{c}\text { Medication } \\
\text { costs (USD) }\end{array}$ & $\begin{array}{c}\text { Healthcare } \\
\text { utilization } \\
\text { costs (USD) }\end{array}$ & $\begin{array}{c}\text { Total direct } \\
\text { treatment } \\
\text { costs (USD) }\end{array}$ & $\begin{array}{c}\text { \% Savings in } \\
\text { Total cost by } \\
\text { FP/Salm }\end{array}$ \\
\hline AHEAD & FP/Salm & 231.65 & 9.20 & 240.85 & $44 \%$ \\
\hline COMPASS & MART & 421.07 & 10.04 & 431.11 & \\
\hline COSMOS & FP/Salm & 127.78 & 30.87 & 158.65 & $47 \%$ \\
\hline MART & 280.48 & 20.20 & 300.68 & \\
\hline Overall & FP/Salm & 186.61 & 12.46 & 199.05 & $43 \%$ \\
\hline & MART & 340.05 & 8.82 & 348.88 & \\
\hline & FP/Salm & 182.01 & 17.51 & 199.53 & $45 \%$ \\
\hline
\end{tabular}

Data are calculated as cost/patient/year.

${ }^{*}$ Percent saving total cost by FP/Salm was calculated by [(total treatment cost MART - total treatment cost FP/Salm)/total treatment cost MART] $\times 100$

${ }^{*}$ data are presented as mean

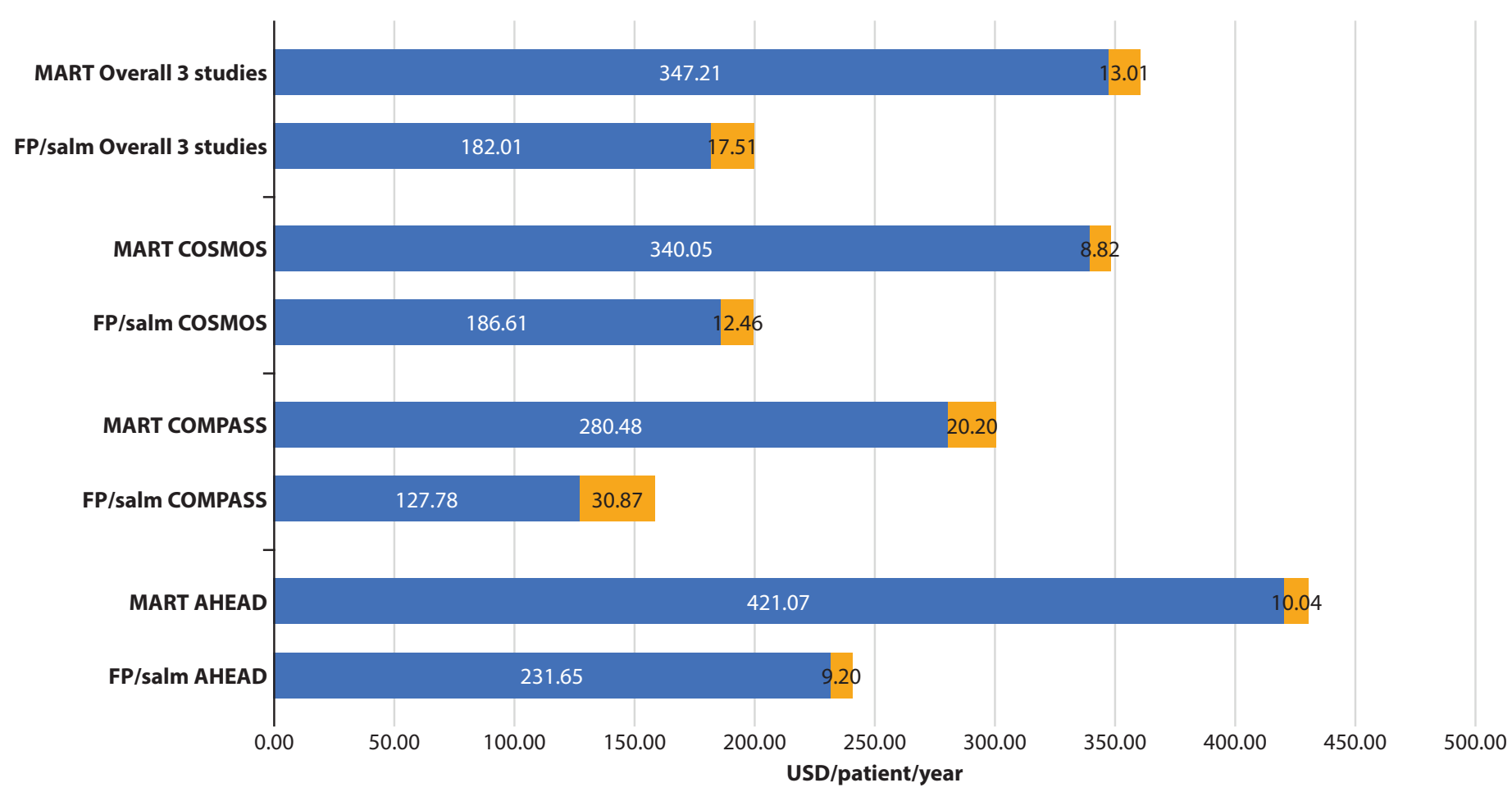

Medication Costs (USD/patient/year)

Healthcare utilization costs (USD/patient/year)

Figure 1. Total direct treatment cost by treatment options in each study

\section{Discussion}

Regular twice-daily FP/Salm with as-needed SABA associated with lower annual total direct treatment cost than BUD/ Form MART in moderate/severe asthma patients when calculated with available data of current medication and healthcare utilization cost in Thailand. Percent saving of regular twice-daily FP/Salm with as-needed SABA was almost half compared to BUD/Form MART. These findings are consistent across all 3 studies.
The results of our study may help physicians and decision makers to understand the differences of cost between different strategies for moderate/severe asthma management in Thailand. In a recent survey of physicians in Asia, in addition to treatment guidelines and physician's personal experience, patient affordability of treatment was identified as an important factor influencing the choice of treatment by physicians for patients with asthma and co-existent rhinitis. ${ }^{20}$ 
Costs of asthma management can be affected by various factors, including new treatment options, country policy, and price change. ${ }^{9}$ Costs of prescription medications and healthcare visits account for the bulk of the costs of asthma. ${ }^{9}$ One of the reasons in some physicians to prescribe MART is saving medication cost from 2 devices (ICS/LABA with as-needed $\mathrm{SABA}$ ) to single device (BUD/Form MART). However, the results of cost comparison can be different between the continents. Our findings are contrast to the previous publications comparing cost between FP/Salm vs MART in resource-rich countries including Canada, Denmark, and Finland as they found that BUD/Form MART was less expensive than FP/ Salm. ${ }^{10-12}$ This is explained by difference of the costs between $\mathrm{FP} / \mathrm{Salm}+$ as needed SABA vs BUD/Form MART between countries. In Table 2, cost of reliever therapy between two treatment strategies are much different; cost per puff of as needed BUD/Form $(160 / 4.5 \mu \mathrm{g})$ is more than 24 times higher than SABA (0.24 vs $0.01 \mathrm{USD} /$ puff, respectively). The annual total direct treatment cost of FP/Salm with as-needed SABA in all 3 studies ${ }^{14-16}$ was lower than BUD/Form MART mainly due to lower medication cost. However, number of healthcare utilization was relatively low in all 3 studies for both treatment strategies. ${ }^{14-16}$

Asthma is a chronic inflammation disease of airway, therefore, the core treatment is anti-inflammatory medication of ICS/LABA for asthma patient step 3-5. ${ }^{1} \mathrm{FP} / \mathrm{Salm}$ was previously reported to be significantly superior to BUD/Form in reducing the rate of moderate/severe exacerbations with sustained regular treatment. ${ }^{21}$ Regular FP/Salm had 57\% lower rate of moderate/severe exacerbations than regular BUD/Form at week 17-24 $(p=0.006)$ in patients with persistent asthma. ${ }^{21}$ ICSs are not therapeutically similar and have different pharmacodynamic and pharmacokinetic properties. ${ }^{22}$ Potency of corticosteroid is usually measured as binding affinity at the glucocorticoid receptor affinities compared with dexamethasone. ${ }^{23}$ Fluticasone propionate (FP) has higher corticosteroid receptor affinity 18 vs 9.4 of Budesonide..$^{23} \mathrm{FP}$ has higher therapeutic index compared to Budesonide. ${ }^{22}$ In addition, for safety concern, FP has lower oral bioavailability, less systemic exposure, than Budesonide. ${ }^{23}$ The efficacy and safety of regular maintenance therapy with the ICS/LABA combination $\mathrm{FP} /$ Salm has been well-established in patients with moderate to severe asthma (both with and without a previous history of asthma exacerbations), demonstrating benefits in terms of achieving and maintaining asthma control, reducing exacerbations, and in improving asthma symptoms and health-related quality of life (HRQL). $5,21,24,25$

MART treatment regimen with the combination of Budesonide and Formoterol has well-described efficacy in reducing the risk of severe exacerbations in studies of patients with moderate asthma and a history of exacerbations. ${ }^{26,27}$ Some studies have shown a lower risk of exacerbations with MART vs conventional best practice ${ }^{26}$ or regular maintenance ICS/LABA therapy ${ }^{27}$ although this is not a consistent observation. ${ }^{8}$ With respect to other asthma outcomes, studies comparing the two treatment regimens have shown similar improvements ${ }^{1,8,26}$ and in studies of patients with no specified exacerbation history, none have demonstrated a reduction in the time to first serious exacerbation with MART versus conventional best practice treatment. ${ }^{28}$

Majority of patients with asthma had poor perception of their asthma symptoms, and over $80 \%$ of uncontrolled asthma patients, per GINA criteria, reported that they perceived their asthma as controlled. ${ }^{29}$ Results from randomized study using MART which aim to use BUD/Form as both controller and reliever may not directly imply to real-world clinical practice because of prescriber confusion; 91 of 173 patients (53\%) receiving MART in UK were additionally dispensed SABA. ${ }^{30}$ MART may provide inadequate anti-inflammatory or undertreatment in some patients. Pavord ID et al. reported patients using BUD/Form MART had increased eosinophils in sputum and bronchial biopsy but in patients using regular fixed-dose ICS/LABA these inflammatory markers were decreased. ${ }^{31}$

In the three studies that could be included in this analysis, differences in yearly exacerbation rates were very small but were significantly in favor of the MART strategy. ${ }^{14-16}$ Even in these studies where MART was associated with lower exacerbation rates, direct healthcare costs were lower with FP/ Salm. Other studies comparing MART with conventional best practice have shown a more mixed picture ${ }^{8,26,28,32,33}$ with some not showing a statistically significant difference in the time to first severe exacerbation (primary endpoint) or difference in exacerbation rates between the two strategies. ${ }^{32,33}$

In a pooled analysis of six open label studies in patients with moderate asthma but no history of exacerbations, none of the included studies showed a statistically significant difference between MART and conventional best practice in the time to first severe exacerbation. ${ }^{28}$ A recent meta-analysis by Rogliani et al reported, in patients with moderate-severe asthma, that high-dose ICS/LABA + as-needed SABA and low-dose to medium-dose MART were equally effective in reducing the risk of severe asthma exacerbation. ${ }^{34}$ In summary, looking at the broad range of clinical studies, superior benefit of MART is not consistently demonstrated, so the cost advantage of FP/Salm reported in this analysis may also apply more widely, depending of course on local medication and treatment costs. With regard to other clinical endpoints, in the AHEAD, COMPASS and COSMOS studies, FP/Salm and MART resulted in similar improvements in asthma symptoms, asthma control scores and health-related quality of life (HRQL), and similar findings have been reported elsewhere for patients with moderate to severe asthma. ${ }^{14-16,34}$

Our study had some important limitations. Firstly, the data was calculated by using medication and healthcare utilization cost from Thai government, therefore, it's not reflected cost in private hospital which is generally higher price than government hospital. Secondly, the data was based on using medication and healthcare utilization cost from 3 randomized control trials, so it may not represent the real-life practice. Finally, this looked at only direct healthcare costs and it would be important in future studies to look at both direct and indirect healthcare costs.

In conclusion, in moderate/severe asthma patients, total direct treatment costs with regular twice-daily $\mathrm{FP} / \mathrm{Salm}$ with as-needed SABA were lower in Thailand than with BUD/Form MART primarily due to lower medication costs. 
This information could be used to make informed decisions about budget allocation in for asthma patient care in Thailand.

\section{Acknowledgement}

This analysis was funded by GlaxoSmithKline (GSK); study no. 213963. The results of this analysis were presented as a poster presentation at Thai Asthma Council (TAC) meeting 11-12 Mar 2021, Bangkok.

\section{Conflict of interest declaration}

TB, JK are employees of GSK. WB has received personal fees from AstraZeneca Thailand, Boehringer Ingelheim and Thai Otsuka. BA is an employee of GSK and holds shares. There are no other conflicts of interest to declare.

\section{References}

1. Global Initiative for Asthma [Internet]. Fontana: Global Initiative for Asthma; c2021 [cited 2020 Apr 15]. Global Strategy for Asthma Management and Prevention; [about 1 screen]. Available from: https// www.ginaasthma.org

2. Vichyanond P, Sunthornchart S, Singhirannusorn V, Ruangrat S, Kaewsomboon S, Visitsunthorn N. Prevalence of asthma, allergic rhinitis and eczema among university students in Bangkok. Respir Med. 2002; 96(1):34-8.

3. Uthaisangsook S. Prevalence of asthma, rhinitis, and eczema in the university population of Phitsanulok, Thailand. Asian Pac J Allergy Immunol. 2007;25(2-3):127-32.

4. Boonsawat W, Charoenphan P, Kiatboonsri S, Wongtim S, Viriyachaiyo V, Pothirat C, et al. Survey of asthma control in Thailand. Respirology. 2004;9(3):373-8.

5. Bateman ED, Boushey HA, Bousquet J, Busse WW, Clark TJ, Pauwels RA, et al. Can guideline-defined asthma control be achieved? The Gaining Optimal Asthma ControL study. Am J Respir Crit Care Med. 2004;170(8): 836-44.

6. Cloutier MM, Dixon AE, Krishnan JA, Lemanske RF, Jr., Pace W, Schatz M. Managing Asthma in Adolescents and Adults: 2020 Asthma Guideline Update From the National Asthma Education and Prevention Program. JAMA. 2020;324(22):2301-17.

7. Bateman ED, Reddel HK, Eriksson G, Peterson S, Ostlund O, Sears MR, et al. Overall asthma control: the relationship between current control and future risk. J Allergy Clin Immunol. 2010;125(3):600-8, 8 e1-8 e6.

8. Chapman KR, Barnes NC, Greening AP, Jones PW, Pedersen S. Single maintenance and reliever therapy (SMART) of asthma: a critical appraisal. Thorax. 2010;65(8):747-52.

9. Nurmagambetov T, Kuwahara R, Garbe P. The Economic Burden of Asthma in the United States, 2008-2013. Ann Am Thorac Soc. 2018;15(3): 348-56.

10. Miller E, Sears MR, McIvor A, Liovas A. Canadian economic evaluation of budesonide-formoterol as maintenance and reliever treatment in patients with moderate to severe asthma. Can Respir J. 2007;14(5):269-75.

11. Wickstrom J, Dam N, Malmberg I, Hansen BB, Lange P. Cost-effectiveness of budesonide/formoterol for maintenance and reliever asthma therapy in Denmark--cost-effectiveness analysis based on five randomised controlled trials. Clin Respir J. 2009;3(3):169-80.

12. Tamminen K, Laine J, Soini E, Martikainen J, Kankaanranta H. Cost -effectiveness analysis of budesonide/formoterol maintenance and reliever therapy versus fixed combination treatments for asthma in Finland ${ }^{*}$. Curr Med Res Opin. 2008;24(12):3453-61.

13. Boonsawat W. Cost-effectiveness of budesonide/formoterol maintenance and rescue therapy in Thailand. Asian Biomed. 2010; 4:571-8

14. Bousquet J, Boulet LP, Peters MJ, Magnussen H, Quiralte J, Martinez -Aguilar NE, et al. Budesonide/formoterol for maintenance and relief in uncontrolled asthma vs. high-dose salmeterol/fluticasone. Respir Med. 2007;101(12):2437-46.

15. Kuna P, Peters MJ, Manjra AI, Jorup C, Naya IP, Martinez-Jimenez NE, et al. Effect of budesonide/formoterol maintenance and reliever therapy on asthma exacerbations. Int J Clin Pract. 2007;61(5):725-36.
16. Vogelmeier C, D'Urzo A, Pauwels R, Merino JM, Jaspal M, Boutet S, et al. Budesonide/formoterol maintenance and reliever therapy: an effective asthma treatment option? Eur Respir J. 2005;26(5):819-28.

17. Aggarwal B, Jones PW, Yunus F, Lan LTT, Boonsawat W, Ismaila A, Ascioglu S. Direct healthcare costs associated with management of asthma: comparison of two treatment regimens in Indonesia, Thailand and Vietnam. J Asthma. 2021;1-8.

18. National drug information, Thailand [Internet]. National drug information, Thailand version dated 9 Dec 2019 [cited 2020 Jul 15]. Available from: http://ndi.fda.moph.go.th/drug_value/index/public/.

19. Health Intervention and Technology Assessment Program (HITAP) [Internet]. Ministry of Public Health, Thailand 2010 [cited 2020 Jul 15] Costing menu, Health Intervention and Technology Assessment Program, Ministry of Public Health, Thailand [about 1 screen]. Available from: http://costingmenu.hitap.net/.

20. Aggarwal B, Shantakumar S, Hinds D, Mulgirigama A. Asia-Pacific Survey of Physicians on Asthma and Allergic Rhinitis (ASPAIR): physician beliefs and practices about diagnosis, assessment, and treatment of coexistent disease. J Asthma Allergy. 2018;11:293-307.

21. Dahl R, Chuchalin A, Gor D, Yoxall S, Sharma R. EXCEL: A randomised trial comparing salmeterol/fluticasone propionate and formoterol/ budesonide combinations in adults with persistent asthma. Respir Med. 2006;100(7):1152-62.

22. Daley-Yates P, Brealey N, Thomas S, Austin D, Shabbir S, Harrison T, et al Therapeutic index of inhaled corticosteroids in asthma: A dose-response comparison on airway hyperresponsiveness and adrenal axis suppression. Br J Clin Pharmacol. 2021;87(2):483-93.

23. Kelly HW. Comparison of inhaled corticosteroids: an update. Ann Pharmacother. 2009;43(3):519-27.

24. Jenkins C, Woolcock AJ, Saarelainen P, Lundback B, James MH. Salmeterol/ fluticasone propionate combination therapy $50 / 250$ microg twice daily is more effective than budesonide 800 microg twice daily in treating moderate to severe asthma. Respir Med. 2000;94(7):715-23.

25. Woodcock AA, Bagdonas A, Boonsawat W, Gibbs MR, Bousquet J, Bateman ED, et al. Improvement in asthma endpoints when aiming for total control: salmeterol/fluticasone propionate versus fluticasone propionate alone. Prim Care Respir J. 2007;16(3):155-61.

26. Cates CJ, Karner C. Combination formoterol and budesonide as maintenance and reliever therapy versus current best practice (including inhaled steroid maintenance), for chronic asthma in adults and children. Cochrane Database Syst Rev. 2013(4):CD007313.

27. Kew KM, Karner C, Mindus SM, Ferrara G. Combination formotero and budesonide as maintenance and reliever therapy versus combination inhaler maintenance for chronic asthma in adults and children. Cochrane Database Syst Rev. 2013(12):CD009019.

28. Demoly P, Louis R, Soes-Petersen U, Naya I, Carlsheimer A, Worth H, et al. Budesonide/formoterol maintenance and reliever therapy versus conventional best practice. Respir Med. 2009;103(11):1623-32.

29. Price D, Fletcher M, van der Molen T. Asthma control and management in 8,000 European patients: the REcognise Asthma and LInk to Symptoms and Experience (REALISE) survey. NPJ Prim Care Respir Med. 2014;24:14009.

30. DiSantostefano RL, Boudiaf N, Stempel DA, Barnes NC, Greening AP. The frequency of, and adherence to, single maintenance and reliever therapy instructions in asthma: a descriptive analysis. NPJ Prim Care Respir Med. 2016;26:16038.

31. Pavord ID, Jeffery PK, Qiu Y, Zhu J, Parker D, Carlsheimer A, et al Airway inflammation in patients with asthma with high-fixed or low-fixed plus as-needed budesonide/formoterol. J Allergy Clin Immunol. 2009;123(5):1083-9, 9 e1-7.

32. Louis R, Joos G, Michils A, Vandenhoven G. A comparison of budesonide/ formoterol maintenance and reliever therapy vs. conventional best practice in asthma management. Int J Clin Pract. 2009;63(10):1479-88.

33. Sears MR, Boulet LP, Laviolette M, Fitzgerald JM, Bai TR, Kaplan A, et al. Budesonide/formoterol maintenance and reliever therapy: impact on airway inflammation in asthma. Eur Respir J. 2008;31(5):982-9.

34. Rogliani P, Ritondo BL, Ora J, Cazzola M, Calzetta L. SMART and as-needed therapies in mild-to-severe asthma: a network meta-analysis. Eur Respir J. 2020;56(3).

35. Puranitee P, Kamchaisatian W, Manuyakorn W, Vilaiyuk S, Laecha O, Pattanaprateep O, et al. Direct medical cost of Thai pediatric asthma management: a pilot study. Asian Pac J Allergy Immunol. 2015;33(4): 296-300. 